\title{
Article
}

\section{Spectroscopic and quartz crystal microbalance (QCM) characterisation of protein-based MIPs}

\author{
El-Sharif, H.F., Aizawa, H. and Reddy, Subrayal M
}

Available at http://clok.uclan.ac.uk/14368/

El-Sharif, H.F., Aizawa, H. and Reddy, Subrayal M ORCID: 0000-0002-7362-

$184 X$ (2015) Spectroscopic and quartz crystal microbalance (QCM)

characterisation of protein-based MIPs. Sensors and Actuators, B: Chemical, 206 . pp. 239-245. ISSN 0925-4005

It is advisable to refer to the publisher's version if you intend to cite from the work.

http://dx.doi.org/10.1016/j.snb.2014.09.053

For more information about UCLan's research in this area go to http://www.uclan.ac.uk/researchgroups/ and search for <name of research Group>.

For information about Research generally at UCLan please go to http://www.uclan.ac.uk/research/

All outputs in CLoK are protected by Intellectual Property Rights law, including Copyright law. Copyright, IPR and Moral Rights for the works on this site are retained by the individual authors and/or other copyright owners. Terms and conditions for use of this material are defined in the policies page.

\section{CLoK}

Central Lancashire online Knowledge www.clok.uclan.ac.uk

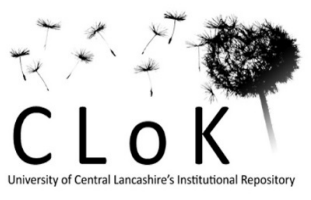


$6 \quad{ }^{1}$ Department of Chemistry, Faculty of Engineering and Physical Sciences, University of

7 Surrey, Guildford, Surrey, GU2 7XH, UK

8

Spectroscopic and Quartz Crystal Microbalance (QCM) Characterization of Protein-

\section{based MIPs}

Hazim F. EL-Sharif ${ }^{1}{ }^{1}$ Hidenbou Aizawa ${ }^{2}$, Subrayal M. Reddy ${ }^{1 *}$

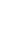

${ }^{2}$ Institute for Environmental Management Technology, National Institute of Advanced Industrial Science and Technology (AIST), 1-1 Higashi, Tsukuba 305-8565, Japan

\section{*Corresponding Author}

\section{Tel : +44 (0) 1483686396, s.reddy@surrey.ac.uk}

\section{Abstract}

We have studied acrylamide-based polymers of varying hydrophobicity (acrylamide, AA; Nhydroxymethylacrylamide, NHMA; N-isopropylacrylamide, NiPAm) for their capability of imprinting protein. Rebinding capacities (Q) from spectroscopic studies were highest for bovine haemoglobin (BHb) MIPs based on AA, Q = 4.8 $\pm 0.21<$ NHMA, Q $=4.3 \pm 0.32<$ NiPAm, $Q=3.6 \pm 0.45$, while also demonstrating low selectivities for non-template proteins ( $<30 \pm 5 \%$ ), with the exception of bovine serum albumin (BSA, $>76 \pm 0.5 \%$ ). When applied to the QCM sensor as thin-film MIPs, NHMA MIPs were found to exhibit best discrimination between MIP and non-imprinted control polymer (NIP) in the order of NiPAm $<$ AA $<$ 
23 NHMA. The extent of template removal and rebinding, using both crystal impedance and

24 frequency measurements, demonstrated that 10\% (w/v):10\% (v/v) sodium dodecyl

25 sulphate:acetic acid ( $\mathrm{pH}$ 2.8) was efficient at eluting template BHb (with $80 \pm 10 \%$ removal).

26 Selectivity studies of NHMA BHb-MIPs revealed higher adsorption and selective recognition

27 properties to $\mathrm{BHb}(64.5 \mathrm{kDa})$ when compared to non-cognate BSA (66 kDa), myoglobin

28 (Mb, $17.5 \mathrm{kDa})$, lysozyme (Lyz, $14.7 \mathrm{kDa}$ ) thaumatin (Thau, $22 \mathrm{kDa}$ ) and trypsin (Tryp, 22.3

$29 \mathrm{kDa}$ ). The QCM gave frequency shifts of $\sim 1500 \pm 50 \mathrm{~Hz}$ for template BHb rebinding in both

30 AA and NHMA MIPs, whereas AA-based MIPs exhibited an interference signal of $\sim 2200 \pm$

$3150 \mathrm{~Hz}$ for non-cognate BSA in comparison to a $~ 500 \pm 50 \mathrm{~Hz}$ shift with NHMA MIPs. Our

32 results show that NHMA-based hydrogel MIP are superior to AA and NIPAM..

33

34 Keywords: Molecular imprinted polymer (MIP); Hydrogel; Protein; Biosensor; QCM 


\section{Introduction}

\section{Introduction}

In recent years, molecularly imprinted polymers (MIPs) have allowed selective extractions that rival immunoaffinity-based separations, and have shown clear advantages over real antibodies for sensor applications: they are easy to fabricate, intrinsically stable, robust, and are able to operate in extreme environments [1], [2] and [3]. MIPs could provide an alternative, inexpensive, fast, and efficient diagnostic method for highly sensitive analytical procedures within the pharmaceutical area [3].

When imprinting complex bio-macromolecules some of the most significant drawbacks in MIP technology are the unprecedented degree of influence that the variation in $\mathrm{pH}$ [4], ionic strength and local matrix effects all have on the gel properties [5], [6], [7] and [8]. This can affect the three dimensional shape and chemical characteristics of the template molecule during polymerisation. This is particularly true when imprinting large bio-macromolecules such as proteins. Proteins are relatively labile, and have variable conformations which are sensitive to solvent environments, $\mathrm{pH}$ and temperature, all of which present a variety of challenges [5]. It has been thought that low imprinting capacities associated with biomacromolecules could be caused by the use of charged functional monomers causing nonspecific electrostatic interactions [5]. Moreover, as with antibodies, MIPs have also shown a degree of cross-selectivity, in that they can bind molecules similar to the native template and cause non-specific binding. It is thought that this is due in part to an excess of functional monomer molecules being randomly distributed and frozen within the imprinted cavity during polymerisation that have an affinity for non-template molecules [3] and [9]. Thus, more sophisticated monomers capable of forming better, stronger and more stable 
interactions that offer better positioning and complementary functionality are widely being sought. Once these parameters are optimised, application to biosensors and analysis of actual biological samples would be more realistic [6], [10], [11] and [12].

Biosensors for proteins are currently expensive to develop because they require the use of expensive antibodies [3] and [13]. However, as MIPs are becoming more and more promising as viable alternatives to natural receptors new MIP-based sensor strategies are being developed [3]. The main advantage of biosensors is the ability to sample outside the laboratory environment with minimal user input. One important part of bio-sensing is transducers, which monitor the reaction between bio-selector and analyte. Among various physical transducers (electrochemical, peizoelectric, etc.), mass sensitive devices such as surface acoustic wave (SAW), surface plasmon resonance (SPR) and quartz crystal microbalance (QCM) have become popular for sensing applications [14], [15], [16] and [17].

Following the thorough analysis of QCM systems for use in fluids over the past 2 decades, this has allowed for more esoteric applications including bio-sensing [16]. In most cases quartz resonators are integrated to oscillator circuits to form a QCM for micro weighing applications. Normally, an equivalent circuit model is fitted to the impedance curve, and the obtained parameters can be used for calculating the resonant frequency and dissipation (D) of the quartz crystal i.e. mass and viscoelastic properties of the deposited layers [15] and [16]. Determining the impedance curve has many advantages; first and foremost it has expanded the range of measurable parameters from rigid thin films, to biologically relevant films of “soft” viscoelastic material. These QCM couplings have been widely used for biomaterials and biosensor studies [10], [12], [16], [18] and [19], where surface confined bio-molecular 
interactions have provided an insight into dissolution of polymer coatings, DNA hybridisation, cell response to pharmacological substances, molecular interactions of drugs and their delivery. The QCM has also been utilised as an immunosensor, where analytes are recognised by antibodies, which are immobilised on a thin layer deposed on a crystal surface. Resulting mass changes are transformed into an electronically measurable quantity. The objective behind the majority of QCM research is to use sensor technology to develop a rapid method for the measurement of bio-molecular affinity reactions, and an in-depth analysis of electrochemical deposition, adsorption and reaction mechanisms of polymers coated on electrodes as ‘thin films' [10], [12], [18], [19] and [20].

The focus of this paper is the tailoring of QCM electrode surface chemistry (i.e. specialised polymer coatings), with a view that these devices can discriminate proteins for bio-sensing and basic surface-molecular interaction studies. In this work, we demonstrate the application of the QCM technique to distinguish between the behaviour of MIPs and NIPs in the presence of cognate and non-cognate proteins. Bovine haemoglobin (BHb, $64.5 \mathrm{kDa}$ ) was chosen as a model protein for its well-known function in the vascular system as a carrier of oxygen, also in aiding the transport of carbon dioxide and regulating blood $\mathrm{pH}$ [3] and [13]. Bovine serum albumin (BSA, $66 \mathrm{kDa}$ ), a non metalloprotein of similar molecular weight to BHb, served to test the selectivity of the BHb-MIP to BSA compared to template BHb, and was compared across a family of acrylamide-based polymer hydrogels.

\section{Experimental}

2.1. Materials 
107 Acrylamide (AA), N-hydroxymethylacrylamide (NHMA), N-iso-propylacrylamide (NiPAm),

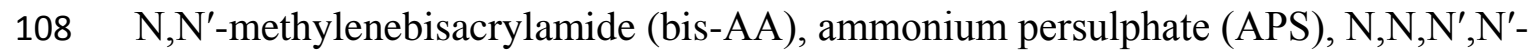
tetramethylethylenediamine (TEMED), sodium dodecyl-sulphate (SDS), glacial acetic acid $(\mathrm{AcOH})$, bovine haemoglobin (BHb), bovine serum albumin (BSA), hen egg-white lysozyme (Lyz), thaumatin from Thaumatococcus danielii (Thau), bovine pancreatic trypsin (Tryp) and equine heart myoglobin (Mb) were all purchased from Sigma-Aldrich, Poole, Dorset, UK. Sieves $(75 \mu \mathrm{m})$ were purchased from Endecotts Ltd. and Inoxia Ltd., UK. AT-cut quartz crystal pieces (9 MHz fundamental resonance) with gold-on-chrome electrodes were supplied by Nihon Dempa Kogyo Company Ltd. (Tokyo, Japan).

\subsection{HydroMIP preparations}

Hydrogel MIPs were synthesised by separately dissolving AA (54 mg), NHMA (77 mg), NiPAm (85.6 mg) and bis-AA as cross-linker (6 mg), (8.5 mg) and (9.5 mg), respectively along with template protein $(12 \mathrm{mg})$ in $960 \mu \mathrm{L}$ of MilliQ water. The solutions were purged

122 with nitrogen for $5 \mathrm{~min}$, followed by an addition of $20 \mu \mathrm{L}$ of a $10 \%(\mathrm{w} / \mathrm{v})$ APS solution and $12320 \mu \mathrm{L}$ of a $5 \%(\mathrm{v} / \mathrm{v})$ TEMED solution. Polymerisation occurred at room temperature (RT, 22 $\left.124 \pm 2{ }^{\circ} \mathrm{C}\right)$ giving total gel densities $(\% \mathrm{~T})$ of $6 \% \mathrm{~T}$, AA/bis-AA (w/v); 8.5\%T, NHMA/bis-AA (w/v); 9.5\%T, NiPAm/bis-AA (w/v), and final crosslinking densities (\%C) of 10\%C (9:1, w/w) for all hydrogels. 
For every MIP created a non-imprinted control polymer (NIP) was prepared in an identical manner but in the absence of protein. After polymerisation, the gels were granulated separately using a $75 \mu \mathrm{m}$ sieve. Of the resulting gels, $500 \mathrm{mg}$ were transferred into $1.5 \mathrm{~mL}$ centrifuge Eppendorf tubes and conditioned by washing with five $1 \mathrm{~mL}$ volumes of MilliQ water followed by five $1 \mathrm{~mL}$ volumes of 10\% (w/v):10\% (v/v) SDS:AcOH (pH 2.8) and another five $1 \mathrm{~mL}$ volume washes of MilliQ water to remove any residual 10\% (w/v):10\% (v/v) SDS:AcOH eluent and equilibrated the gels. Each wash step was followed by a centrifugation, whereby the gels were centrifuged using an Eppendorf mini-spin plus centrifuge for 3 min at 6000 rpm (RCF: $2419 \times$ g). All supernatants were collected for analysis by spectrophotometry to verify the extent of template removal. It should be noted that the last water wash and eluent fractions were not observed to contain any protein. Therefore we are confident that any remaining template protein within the MIPs did not continue to leach out during the rebinding studies.

\subsection{Rebinding studies}

Once the gels (500 mg) were equilibrated, a $1 \mathrm{~mL}$ template protein solution prepared in MilliQ water containing 3 mg of protein was added to the target MIPs and NIP controls and was allowed to associate at RT $\left(22 \pm 2{ }^{\circ} \mathrm{C}\right)$ for $20 \mathrm{~min}$. Selectivity studies were also conducted to assess the relative imprinting factor of the original protein template. This was achieved by loading non-cognate proteins on a BHb imprinted gel. Gels were then washed with four $1 \mathrm{~mL}$ volumes of MilliQ water. Each reload and wash step for all MIPs and NIP controls was followed by centrifugation at 6000 rpm (RCF: $2419 \times$ g) for 3 min. All supernatants were collected for analysis by spectrophotometry. 
2.4. Spectrophotometric analysis

Calibration curves in MilliQ water and 10\% (w/v):10\% (v/v) SDS:AcOH were prepared for BHb, BSA, Lyz, Tryp and Mb. Spectral scans revealed peak wavelengths for BHb in MilliQ water and 10\% (w/v):10\% (v/v) SDS:AcOH to be $405 \mathrm{~nm}$ and $395 \mathrm{~nm}$, respectively. Peak wavelengths for BSA in MilliQ water and 10\% (w/v):10\% (v/v) SDS:AcOH were found to be $288 \mathrm{~nm}$ and $290 \mathrm{~nm}$ respectively. Peak wavelengths for Lyz in MilliQ water and 10\% AcOH:SDS were found to be $291 \mathrm{~nm}$ and $296 \mathrm{~nm}$ respectively. Peak wavelengths for Tryp in

2.5. Quartz crystal microbalance (QCM) analysis of thin film MIPs

QCM crystals were sealed and air capped (single-sided) with PVC glue in-order to prevent short circuiting when the QCM was submerged in solution [10]. Poly AA, NHMA and NiPAm gels for BHb were synthesised using the hydrogel production procedures outlined in Section 2.2. Before polymerisation, MIPs and NIPs were deposited as thin films onto the capped QCM crystals. Thin-films were achieved by beading and compressing $10 \mu \mathrm{L}$ of the polymerising solutions directly onto the crystals. QCM frequency and impedance 
measurements were taken using an Agilent 4194A Impedance Analyser. An in-house written QBasic programme was used to drive the analyser and collect series resonance frequency and impedance data in real time.

2.5.1. Elution and rebinding studies

MIP and NIP polyAA thin-film capped crystals were firstly immersed in MilliQ water, followed by $10 \%(\mathrm{w} / \mathrm{v}): 10 \%(\mathrm{v} / \mathrm{v}) \mathrm{SDS}: \mathrm{AcOH}$ in order to remove imprinted protein primarily from the surface of the polymer. This was followed by another submersion in MilliQ water to remove any residual surfactant and to re-condition the hydrogel. After subsequent stabilisation of the QCM response, template protein was reloaded by immersing the QCM in a $3 \mathrm{mg} / \mathrm{mL}$ BHb solution and the response trace was recorded at $\mathrm{RT}\left(22 \pm 2{ }^{\circ} \mathrm{C}\right)$.

\subsubsection{Selectivity studies}

Continuous real-time scans were conducted in-order to assess characteristic impedance changes of the gels during surface exposure to wash, elute and protein rebinding conditions.

During a typical run, the MIP thin-film capped crystals were submerged sequentially in various solutions such as 10\% (w/v):10\% (v/v) SDS:AcOH, MilliQ water or $3 \mathrm{mg} / \mathrm{mL}$ protein solutions (cognate BHb and non-cognate BSA, Thau, Lyz and Tryp) for a set time of 5 min each, and crystal impedance and frequency responses were recorded at RT ( $\left.22 \pm 2{ }^{\circ} \mathrm{C}\right)$. The latter procedure was followed for AA, NiPAm and NHMA based MIP hydrogels for BHb. 


\subsection{MIP selectivity}

200

The molecular imprinting effect is characterised by the rebinding capacity (Q) of protein to

201 the gel polymer (mg/g) exhibited by the protein-specific MIP and the control NIP, and is calculated using Eq. (1), where $\mathrm{Ci}$ and $\mathrm{Cr}$ are the initial protein and the recovered protein concentrations $(\mathrm{mg} / \mathrm{mL}$ ) respectively (which specifies the specific protein bound within the gel), $\mathrm{V}$ is the volume of the initial solution (mL), and $\mathrm{g}$ is the mass of the gel polymers (g).

$206 \quad Q=\frac{\left[C_{i}-C_{r}\right] V}{g}$

Fig. 1A shows the rebinding capacities and imprinting factors of polyacrylamide (polyAA) MIP and NIPs for several different proteins. The internal measure of the imprinting factor between MIP and NIP serves to demonstrate that the MIP possesses selective cavities for the rebinding of template molecule compared to NIP controls. It can clearly be seen that there is a distinctive rebinding capacity variation for each imprinted protein template within a polyAA MIP. This has previously been attributed to protein size, cross-linking density, and the initial degree of association within the polymer matrix [4].

Gels based on N-hydroxymethylacrylamide (NHMA) exhibited similar rebinding trends, whereas poly-N-isopropylacrylamide gels (polyNiPAm) demonstrated lower rebinding capacities. Thus, bulk gel characterisation revealed the highest rebinding capacities for BHb MIPs based on polyAA $(\mathrm{Q}=4.8 \pm 0.21)$, followed by polyNHMA $(\mathrm{Q}=4.3 \pm 0.32)$, 
$230 k=\frac{\mathrm{IF}_{B H b}}{\mathrm{IF}_{x}}$

polyNiPAm $(\mathrm{Q}=3.6 \pm 0.45)$. These gel imprinting trends are in agreement with those previously published [4], [9] and [10].

Selectivity studies were also conducted to confirm a BHb specific imprinting effect and to assess the relative imprinting factor of cross-selective binding profiles. The cross-reactivity of the BHb-imprinted MIPs for non-cognate proteins was quantified using relative imprinting factors (k), Eq. (2), where IFBHb is the imprinting factor for BHb, and is calculated by IF = $[\mathrm{Ci}-\mathrm{Cr}] \mathrm{MIP} /[\mathrm{Ci}-\mathrm{Cr}] \mathrm{NIP}$, and IFx is the imprinting factor of the cross-reacting noncognate proteins on a BHb MIP. For the template BHb $\mathrm{k}=1$, and for non-cognate proteins that are less-specific for the BHb MIP, $\mathrm{k}<1$.

231 The data (Fig. 1B) suggests that both non-cognate trypsin (Tryp) and lysozyme (Lyz) proteins

232

233 have relatively low affinities for the BHb-specific MIP, $\mathrm{k} \approx 0.2 \pm 0.05$. However, bovine serum albumin (BSA), which is a similar size to BHb, exhibited a high degree of interference binding (cross selectivity) resulting in high $\mathrm{k}$ values of $0.65 \pm 0.05$. Myoglobin $(\mathrm{Mb})$ also exhibited some degree of cross-selectivity; this can be attributed to the size of Mb, which is a quarter that of $\mathrm{BHb}(17.5 \mathrm{kDa})$, and its similarity to a single BHb sub-unit [4]. Interestingly though, when reversed, a polyAA BSA-MIP exposed to non-target BHb protein had relatively low affinity. It would appear that BSA has a high ability to bind non-specifically to a BHb MIP, whereas BHb does not exhibit the same ability within a BSA MIP. 
Competitive binding studies using a 50:50 mixture of BHb:BSA (3 mg/mL total) on a MIP-

242 BHb were also conducted (Fig. 1B). The addition of BSA caused an obvious capacity decrease of BHb binding on the BHb-MIP, suggesting that the rebinding of BHb was displaced by the competing BSA or by protein-protein interactions [21]. As the size, structure, and specificity of the imprinted cavities should be in favour of the BHb template, it is rational that the addition of BSA as a competing protein would not bind to the BHbspecific imprinted cavities. Gai et al. previously demonstrated that BSA does not bind specifically to a BHb MIP, but rather displaces the non-specific recognition sites of cavities and the nonspecific binding of BHb to BHb-MIP [21]. Moreover, although BSA and BHb share similar sizes (66 kDa and $64.5 \mathrm{kDa}$, respectively), it should be noted that BSA has a pI of 4.6 and BHb a pI of (6.8-7.0). Since competitive binding was performed under MilliQ water (pH 5.4), conditions are in favour of BSA [21] and [22]. Above its pI, BSA becomes negatively charged and the groups exist as single bondNH2 and single bondCOO-, this overall negative net charge induces more favourable and complementary hydrogen bonding interactions, resulting in increased specific as well as non-specific binding [4].

\subsection{QCM sensor application of MIPs}

Thin film BHb MIPs were prepared on the surface of a QCM chip and the sensor was exposed sequentially to MilliQ water, 10\% (w/v):10\% (v/v) SDS:AcOH and $3 \mathrm{mg} / \mathrm{mL}$ protein solutions at RT $\left(22 \pm 2{ }^{\circ} \mathrm{C}\right)$. We have previously published on the thickness of the thin films on sensor chips with an average thickness of $138 \pm 9 \mathrm{~nm}$ [6]. Given that for a $9 \mathrm{MHz}$ crystal the shear wave decay length is $250 \mathrm{~nm}$ at RT [23], we are within the sensing region of the QCM to measure both bulk and surface effects within the MIP film. 
Fig. 2A and B shows the QCM impedance and frequency responses following immersion in a solution of $10 \%(\mathrm{w} / \mathrm{v}): 10 \%(\mathrm{v} / \mathrm{v})$ SDS:AcOH in order to remove imprinted protein from the surface of the polymer. Previous investigations have shown that optimum conditions for protein removal of up to $80 \%$ have been achieved using 10\% (w/v):10\% (v/v) SDS:AcOH [9]. Using this acid/surfactant combination the positively charged protein attaches to the negatively charged surface of SDS micelles and disrupts the hydrophobic bonds. Since there is a significant shift in both resonance frequency and impedance it can be assumed that some of the BHb imprinted template has been successfully removed from the MIP.

It is worth noting the two distinct differences in the impedance response when compared with the frequency response. Firstly, the impedance response has much reduced noise in the signal in contrast to the frequency response. Secondly, there are significant additional transitions (e.g. at 350 and $650 \mathrm{~s}$ ) in the signal which are being observed in the $\mathrm{Z}$ response, but not in the frequency response. It has been suggested that whereas the frequency response predominately demonstrates the QCM mass response only within an adlayer, the electrical impedance gives timescale following initial immersion [23], [24] and [25].

After subsequent stabilisation of the QCM response, the template BHb was then reloaded by immersing the QCM in a $3 \mathrm{mg} / \mathrm{mL}$ BHb solution and the response trace recorded. Fig. 2C and D compares the final QCM impedance and frequency responses to template BHb exposure of each MIP and its corresponding NIP. It can be seen that upon addition of a $3 \mathrm{mg} / \mathrm{mL} \mathrm{BHb}$ solution to the BHb MIP caused significant QCM responses compared with NIP thin-film hydrogels. This suggests that MIP thin-film gels are affected by specific binding of target 
BHb. This distinct difference between responses exhibited by MIP and the NIP control strongly supports that binding and elution of target protein gave rise to distinct impedance transitions. The $200 \pm 50 \mathrm{~Hz}$ frequency shift observable by both MIP and NIP during the initial loading step (Fig. 2D) is suggestive of a solution viscosity effect.

Real time impedance response following sequential immersion in solutions of $\mathrm{BHb}, 10 \%$ (w/v):10\% (v/v) SDS:AcOH and BSA were also measured. Three distinct types of responses were observed depending on the acrylamide-based monomer used. The key difference between the polymers is their hydrophilicity dictated by the hydrophilic hydroxyl group in NHMA and the hydrophobic isopropyl group in NiPAm. AA sits between the two in degree of hydrophilicity (polyNHMA > polyAA > polyNiPAm), which agrees with the order of best performance of the polymers as BHb MIPs in previous QCM studies [10]. Fig. 3 compares the final QCM response to cognate and non-cognate protein exposure of each MIP with its corresponding NIP. Interestingly, the NiPAm MIP and NIP both show a near zero frequency response to template $\mathrm{BHb}$ and non-cognate $\mathrm{BSA}$, indicating that NiPAm is equally unselective for both proteins as is the control non-imprinted polymer. The non-response of $\mathrm{NiPAm}$ to either $\mathrm{BHb}$ or BSA suggests that there is a resistance to either protein to bind to the polymer. The striking difference in selectivities between cognate and non-cognate proteins for NHMA and AA suggests that the hydroxyl group in NHMA plays a significant role in the selective binding of BHb and the lack of binding of BSA.

Moreover, variations of the series resonance frequency demonstrated to be highly dependent on the test solution used (Fig. 4A). The impedance data is presented here because in comparison to the frequency response, there is much reduced noise in the signal following each solution phase immersion. It can be seen that MIP thin-films exposed to a 10\%:10\% 
313 (v/v) SDS:AcOH solution exhibited an immediate significant decrease in impedance (500 \pm

$314100 \Omega$ ); this is possibly due to the increase in the viscosity of the solution caused by the presence of SDS micelles in the solution. Moreover, it can clearly be seen that the introduction of non-template BSA also exhibits an impedance response within the poly AA MIP, suggesting some non-specific binding within the BHb-HydroMIP. Thus, there is a high degree of cross-selectivity present for our AA-based MIPs (>70\%), and this interference is absent when NHMA-based MIPs are used (<20\%) as seen in Fig. 3.

To further test the BHb-MIP selectivity, we investigated the rebinding of template BHb after exposing the MIP with non-target BSA. The resulting quantified imprinting effect of BHb for polyAA MIP thin-film gels can be seen in the impedance responses (Fig. 4B). The comparison demonstrates that both HydroMIP and HydroNIP films act differently under water wash, elution and load (solution immersion) treatments following BSA loading. It can be seen that when non-target BSA is loaded first, the QCM impedance response is now negligible. Interestingly, impedance responses are also almost negligible when BHb is introduced after prior exposure to BSA. Although the loaded BSA did not associate specifically with the BHb-MIP thin-film surface, an interesting and lasting effect inhibits $\mathrm{BHb}$ from easily binding to recognition sites. Indeed, BSA is similar to the template BHb in size, but the spatial arrangement of the effective groups on its exterior are different from $\mathrm{BHb}$, and the recognition sites in the BHb-MIP cavities are not complementary in shape to BSA [22]. Therefore, little to no selectivity of BSA on BHb-MIPs should be expected. Therefore, in the case of AA-based MIPs, the inhibition effect is most likely due to the ability of BSA to exhibit protein binding on the MIP surface but not within cavities. Formation of a BSA biolayer above (but not within) the cavities would block subsequent cavity-selective MIP binding for its cognate protein [22]. This is further indication that BHb-MIPs distinguish proteins not only based on molecular size, but also on the synergistic effect of shape memory, 
and multiple weak hydrogen bonding interactions specific to template protein in macromolecular recognition [21], [22] and [26].

Moreover, further studies to interrogate the recognition capabilities of MIPs were carried out using a range of non-metalloproteins chosen for their different sizes and functionalities compared to BHb, BSA and Mb. Of these proteins: lysozyme (Lyz), a glycoside hydrolase enzyme $(14.7 \mathrm{kDa})$ that is part of the innate immune system, and exists as a natural form of protection from pathogens like Salmonella, E. coli, and Pseudomonas [9], [10] and [27]. Thaumatin (Thau), a sweetener or flavour modifier (22 kDa) often used in crystallisation studies due to its ease of use and crystal formation [27]. Trypsin (Tryp), a serine protease enzyme or proteinase ‘digestive enzyme’ (23.8 kDa) commonly imprinted within MIPs [9], [10] and [27]. Fig. 5 shows that the BHb-MIPs based on all three polymers (AA, NHMA, and NiPAm) are essentially non-responsive to the addition of the three smaller proteins Thau, Lyz and Tryp respectively. An average NIP response was calculated based on all three polymers and used as an illustration to demonstrate the negligible responses exhibited by the MIP properties. The negligible responses exhibited by the QCM sensor concur with the qualitative data and confirm that these small proteins exhibit no selective specific/non-specific binding characteristics to a BHb-imprinted MIP.

\section{Conclusions}

A family of acrylamide-based MIPs have been characterised for their imprint efficiency using spectrophotometric and QCM sensor techniques for biosensor development. Varied rebinding capacities and relative imprinting factors have been achieved using bulk characterisation. We 
361 have demonstrated that MIP selectivity is a function of the hydrophilicity of the acrylamide

362 monomer used to form the MIP. Three distinct types of QCM responses were observed

363 depending on the acrylamide used (polyNHMA $>$ polyAA $>$ polyNiPAm), which agrees with

364 the order of best performance of the polymers in previously published QCM studies. The

365 selectivity of BHb-MIP for BHb and BSA was also compared via QCM, along with several

366 other proteins. Results demonstrated BHb-MIP to have better selective adsorption and

367 recognition properties to BHb than BSA when using the hydrophilic NHMA as a MIP

368 polymer matrix. Therefore, the QCM sensor was able to indicate MIP surface activity and

369 provide physical interpretation in terms of hydrophilicity of the polymer matrix that forms the

370 MIP and protein selectivity. Our QCM sensor also has the ability to assess the extent of

371 specific protein binding by sensing surface-specific bound cognate protein to MIPs compared

372 to non-imprint NIP controls. We expect, once fully developed, that the benefits of sensitivity,

373 specificity and stability of MIPs coupled with discriminatory techniques, such as QCM, will

374 be crucial to the future impact of portable diagnostics for personal healthcare and use by

375 health professionals. The technology also presents major potential benefits to environmental

376 and food monitoring.

378 Acknowledgements

379 The authors wish to thank the UK Engineering and Physical Sciences Research Council

380 (EPSRC) Grants (EP/G014299/1) and NERC/ACTF (RSC) for supporting this work. 
Vijayaraghavan, Molecularly imprinted nanopatterns for the recognition of biological warfare agent ricin Biosens. Bioelectron., 25 (2009), pp. 592-598 imprinted polymers Acta Biomater., 8 (2012), pp. 461-473

The rational development of molecularly imprinted polymer-based sensors for protein detection Chem. Soc. Rev., 40 (2011), pp. 1547-1571 molecularly imprinted polymers (HydroMIPs) following buffer conditioning Anal. Chim. Acta, 809 (2014), pp. 155-161

[5] E. Verheyen, J.P. Schillemans, M. van Wijk, M. Demeniex, W.E. Hennink, C.F. van Nostrum Challenges for the effective molecular imprinting of proteins Biomaterials, 32 (2011), pp. 3008-3020

[6] S.M. Reddy, D.M. Hawkins, Q.T. Phan, D. Stevenson, K. Warriner Protein detection using hydrogel-based molecularly imprinted polymers integrated with dual polarisation interferometry Sens. Actuators B: Chem., 176 (2013), pp. 190-197

[7] S.A. Piletsky, N.W. Turner, P. Laitenberger Molecularly imprinted polymers in clinical 401 diagnostics - future potential and existing problems Med. Eng. Phys., 28 (2006), pp. 971-977 of the field Int. J. Pharm., 364 (2008), pp. 188-212 
405 [9] D.M. Hawkins, D. Stevenson, S.M. Reddy Investigation of protein imprinting in 406 hydrogel-based molecularly imprinted polymers (HydroMIPs) Anal. Chim. Acta, 542 (2005), 407 pp. $61-65$

408

[10] S.M. Reddy, Q.T. Phan, H. El-Sharif, L. Govada, D. Stevenson, N.E. Chayen

Protein crystallization and biosensor applications of hydrogel-based molecularly imprinted polymers Biomacromolecules, 13 (2012), pp. 3959-3965 biotech application: selectivity and ruggedness Proc. Eng., 47 (2012), pp. 534-537 enantio-selective analysis of malic acid isomers Sens. Actuators B: Chem., 181 (2013), pp. 596-604 binding in hydrogel-based molecularly imprinted polymersElectrochim. Acta, 56 (2011), pp. 9203-9208

421 biological applications of quartz crystal microbalance Trends Biotechnol., 27 (2009), pp. 689-697

[15] T.M.A. Gronewold Surface acoustic wave sensors in the bioanalytical field: recent trends and challenges Anal. Chim. Acta, 603 (2007), pp. 119-128 
426 [16] U. Latif, S. Can, O. Hayden, P. Grillberger, F.L. Dickert Sauerbrey and anti-Sauerbrey

427 behavioral studies in QCM sensors - detection of bioanalytes Sens. Actuators B: Chem., 176

428 (2013), pp. 825-830

429 [17]C. Steinem, A. Janshoff Sensors: piezoelectric resonators P. Worsfold, A. Townshend,

430 C. Poole (Eds.), Encyclopedia of Analytical Science (2nd ed.), Elsevier, Oxford (2005), pp.

$431 \quad 269-276$

432 [18] K.K. Reddy, K.V. Gobi Artificial molecular recognition material based biosensor for

433 creatinine by electrochemical impedance analysis Sens. Actuators B: Chem., 183 (2013), pp.

$434 \quad 356-363$.

435 [19] K. Reimhult, K. Yoshimatsu, K. Risveden, S. Chen, L. Ye, A. Krozer

436 Characterization of QCM sensor surfaces coated with molecularly imprinted nanoparticles

437 Biosens. Bioelectron., 23 (2008), pp. 1908-1914

438 [20] B. Khadro, C. Sanglar, A. Bonhomme, A. Errachid, N. Jaffrezic-Renault

439 Molecularly imprinted polymers (MIP) based electrochemical sensor for detection of urea

440 and creatinine Proc. Eng., 5 (2010), pp. 371-374

441 [21] Q. Gai, F. Qu, T. Zhang, Y. Zhang The preparation of bovine serum albumin surface-

442 imprinted superparamagnetic polymer with the assistance of basic functional monomer its

443 application for protein separation J. Chromatogr. A, 1218 (2011), pp. 3489-3495

444 [22] Q. Gai, F. Qu, Y. ZhangThe preparation of BHb-molecularly imprinted gel polymers

445 and its selectivity comparison to BHb and BSA Sep. Sci. Technol., 45 (2010), pp. 2394-2399 
447 View Record in Scopus | Full Text via CrossRef | Citing articles (9)

448 [23]K.A. Marx Quartz crystal microbalance: a useful tool for studying thin polymer films and 449 complex biomolecular systems at the solution-surface interface

450 Biomacromolecules, 4 (2003), pp. 1099-1120

451 [24] S. Kurosawa, J. Park, H. Aizawa, S. Wakida, H. Tao, K. Ishihara Quartz crystal

452 microbalance immunosensors for environmental monitoring Biosens. Bioelectron., 22 (2006),

453 pp. 473-481

454 [25] T. Zhou, K.A. Marx, M. Warren, H. Schulze, S.J. Braunhut

455 The quartz crystal microbalance as a continuous monitoring tool for the study of endothelial

456 cell surface attachment and growth Biotechnol. Prog., 16 (2000), pp. 268-277

457 [26] Zhou Xue, He Xi-Wen, Chen Lang-Xing, Li Wen-You, Zhang Yu-Kui

458 Optimum conditions of separation selectivity based on molecularly imprinted polymers of

459 bovine serum albumin formed on surface of aminosilica Chin. J. Anal. Chem., 37 (2009), pp.

$460 \quad 174-180$

461 [27] E. Saridakis, S. Khurshid, L. Govada, Q. Phan, D. Hawkins, G.V. Crichlow, E. Lolis,

462 S.M. Reddy, N.E. Chayen Protein crystallization facilitated by molecularly imprinted

463 polymers Proc. Natl. Acad. Sci., 108 (2011), pp. 11081-11086

464 
Fig. 1.

466 (A) Rebinding capacities $(Q)$ and imprinting factors of $\mathrm{MIP}_{\text {polyAA }}$ and $\mathrm{NIP}_{\text {polyAA }}$ hydrogels for several proteins in MilliQ water media: bovine haemoglobin (BHb), bovine serum albumin (BSA), myoglobin $(\mathrm{Mb})$, lysozyme (Lyz), trypsin (Tryp); (B) relative imprinting factors $(k)$ for a range of $\mathrm{BHb}$ -

469 MIP $_{\text {poyAa }}$ cross-reactants in MilliQ water media. Results illustrate higher MIP selectivities for cognate $\mathrm{BHb}$ and the degree of cross-selectivity for other non-template analytes. Data represents mean \pm S.E.M., $n=3$.

472
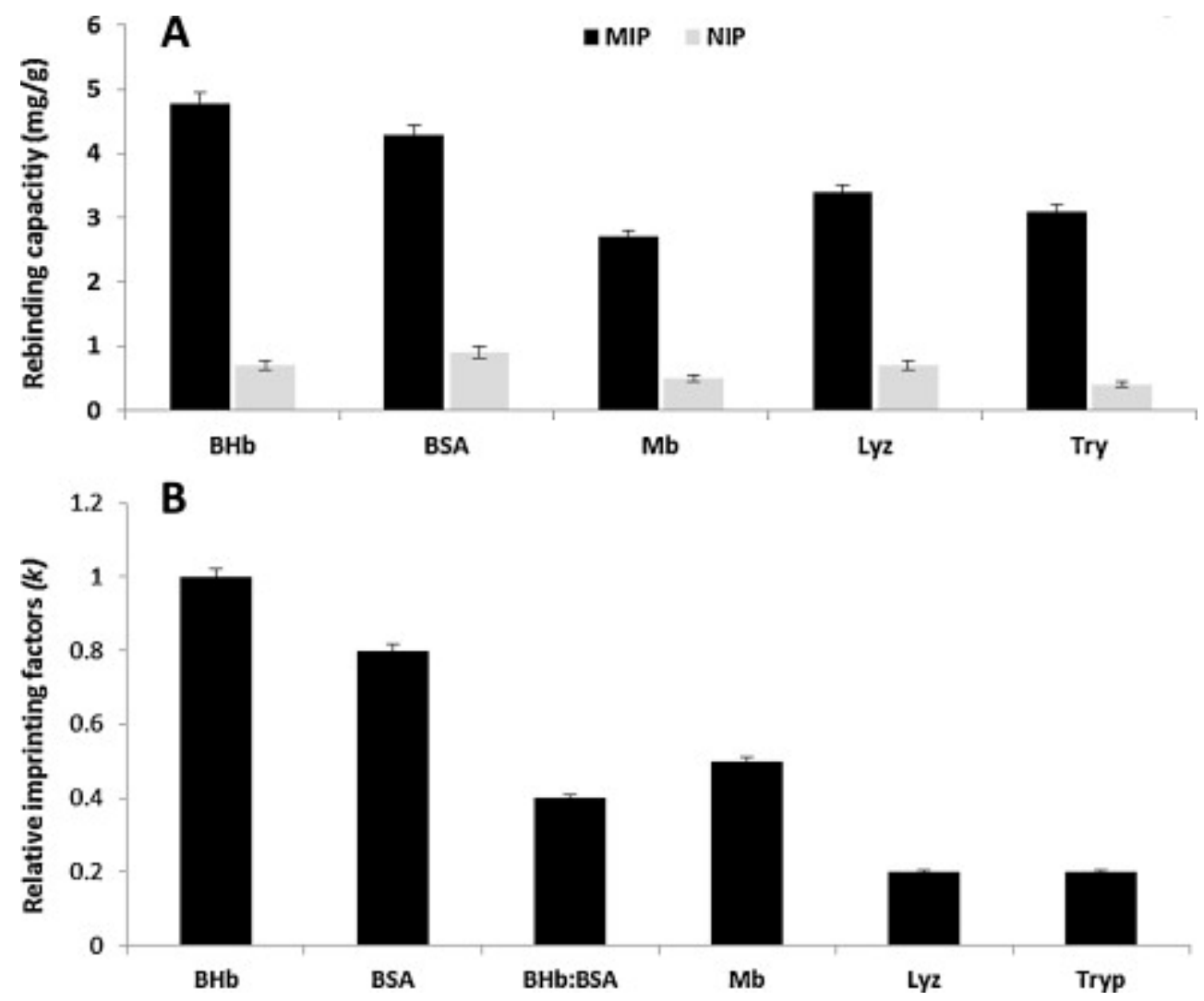

Fig. 2.

QCM response to the immersion of polyAA-BHb hydrogel thin-film MIP in 10\% (w/v):10\% (v/v) SDS:AcOH in order to follow protein elution (arrow indicates time of MIP immersion): (A) impedance $(\Delta Z)$, (B) frequency $(\Delta \mathrm{f})$; QCM responses to $\mathrm{BHb}(3 \mathrm{mg} / \mathrm{mL}$ ) loading onto a BHb imprinted polyAA

478 hydrogel thin-film (arrow indicates time of immersion in protein solution): (C) impedance ( $\Delta Z$ ) and (D) frequency $(\Delta f)$. 

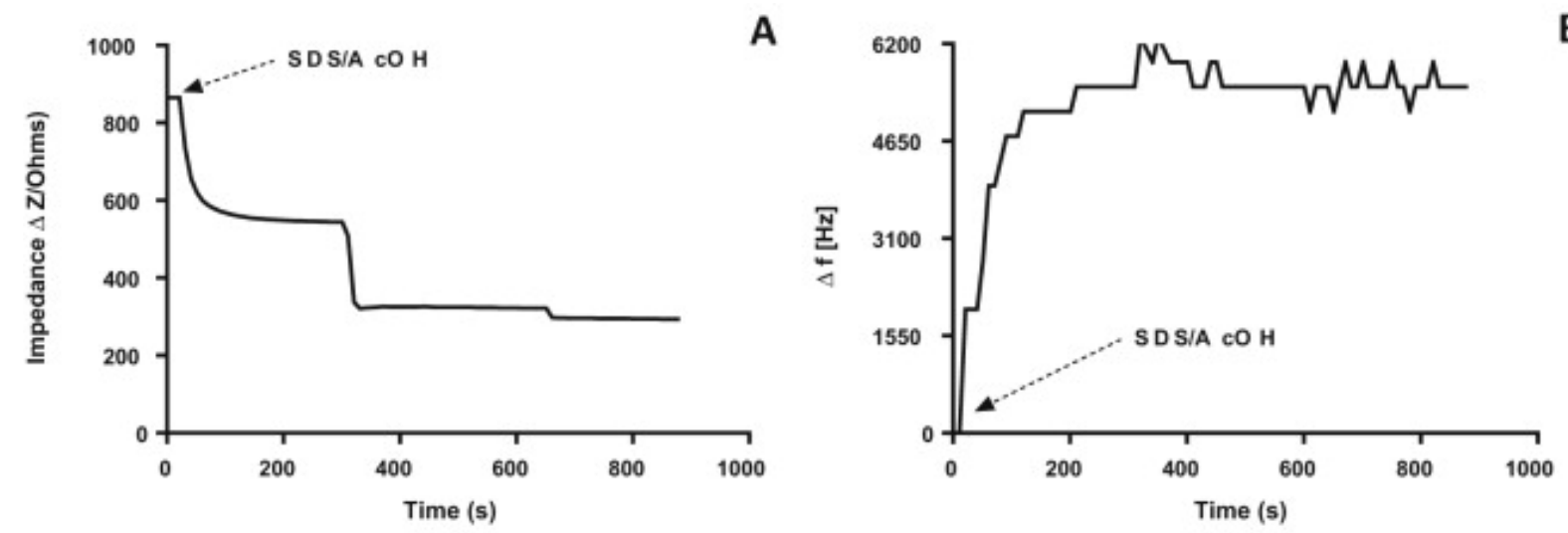

B
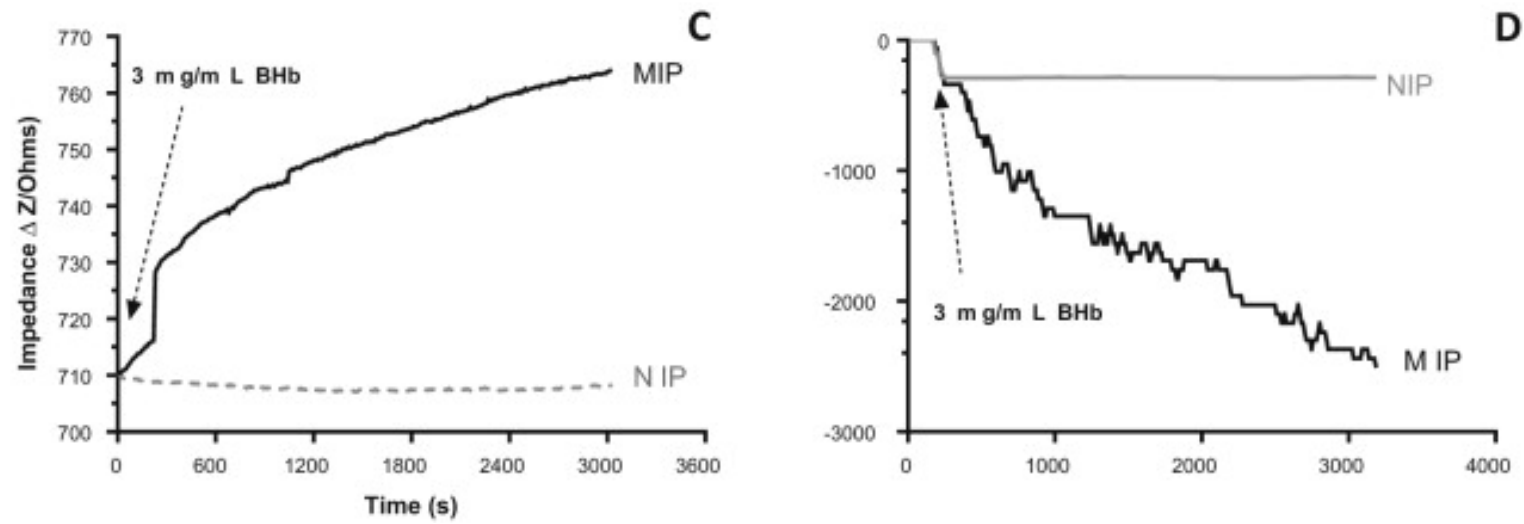

Fig. 3.

QCM frequency shift responses of NiPAm, AA and NHMA polymer MIPs and NIPs to cognate BHb and non-cognate BSA loading $(3 \mathrm{mg} / \mathrm{mL})$ after $5 \mathrm{~min}$ of exposure. Data represents

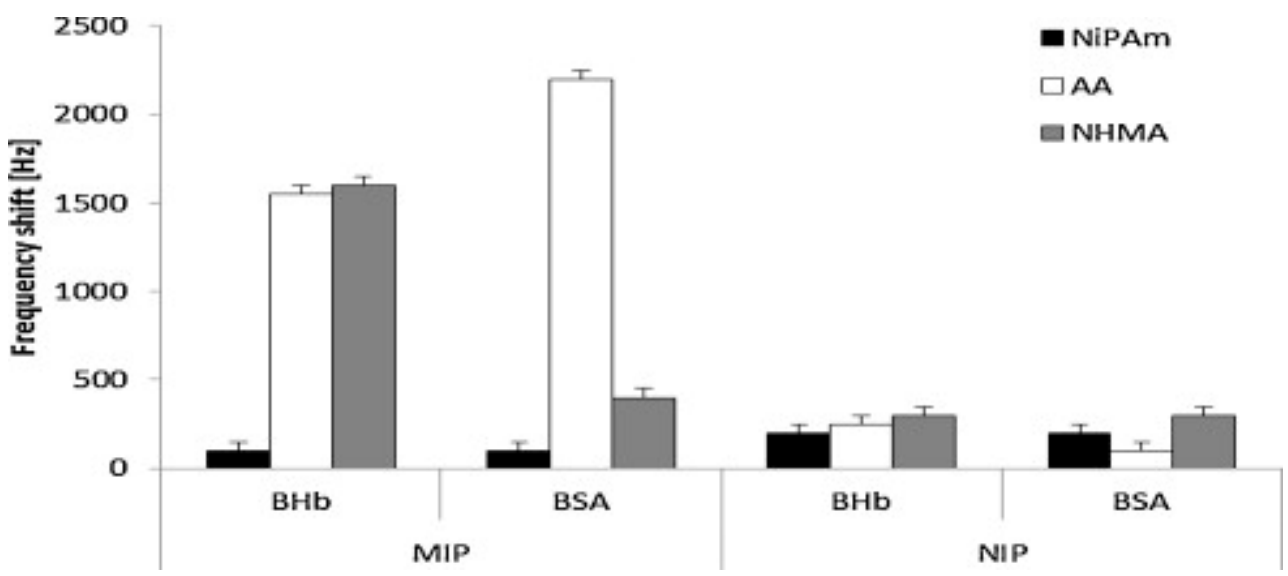

Fig. 4.

491 Real time QCM impedance responses: (A) direct BHb rebinding and BSA cross-selectivity on a BHb$\mathrm{MIP}_{\text {polyA }} ;(\mathrm{B}) \mathrm{BSA}$ cross-selectivity on a BHb-MIP polyA followed by BHb rebinding. 

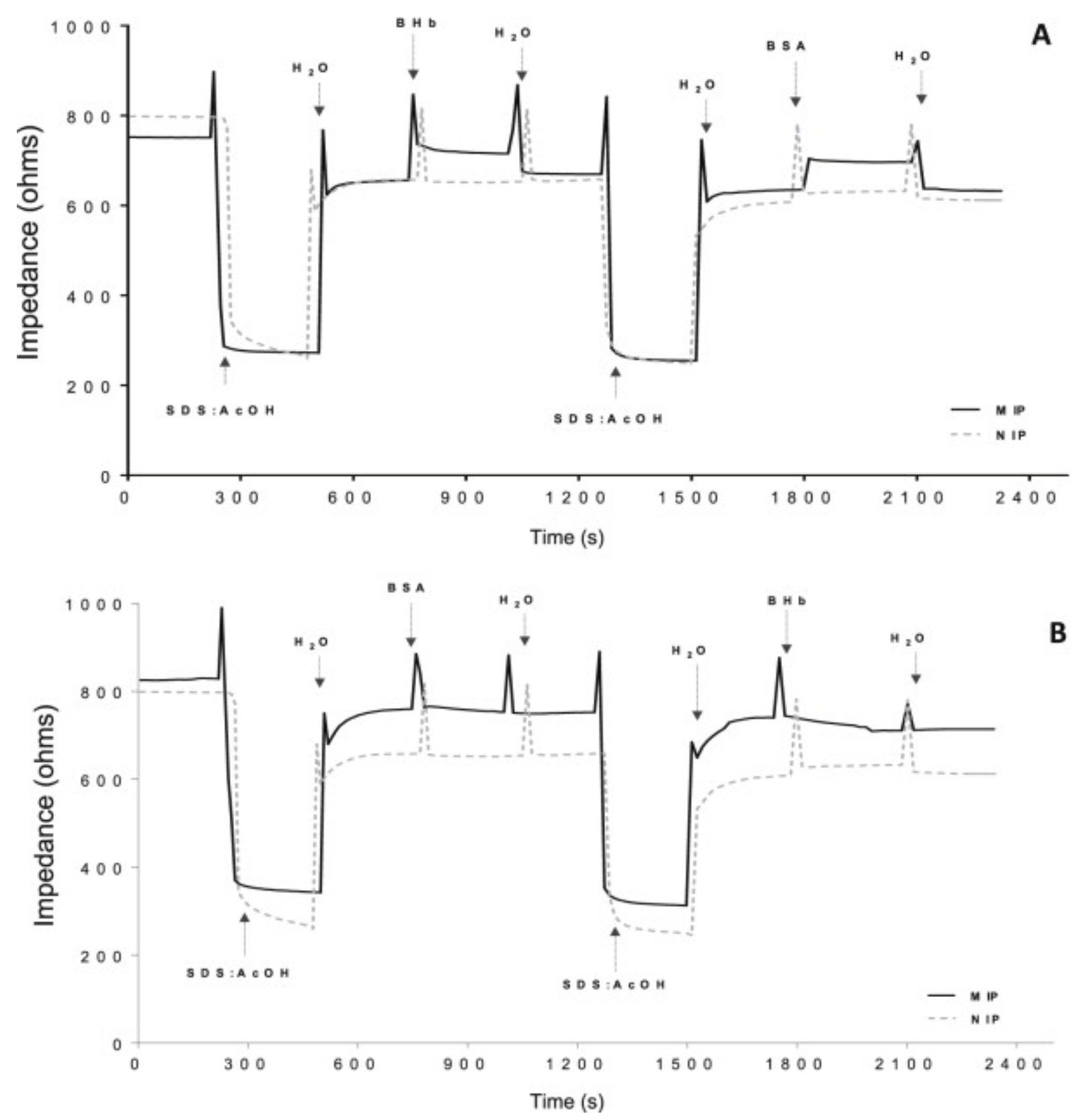

495

496 Fig. 5.

497 QCM response of functionalised acrylamide BHb MIPs to non-cognate proteins thaumatin (Thau), 498 lysozyme (Lyz), and trypsin (Tryp) after $\mathrm{H}_{2} \mathrm{O}$ washes and an SDS:AcOH elute. 


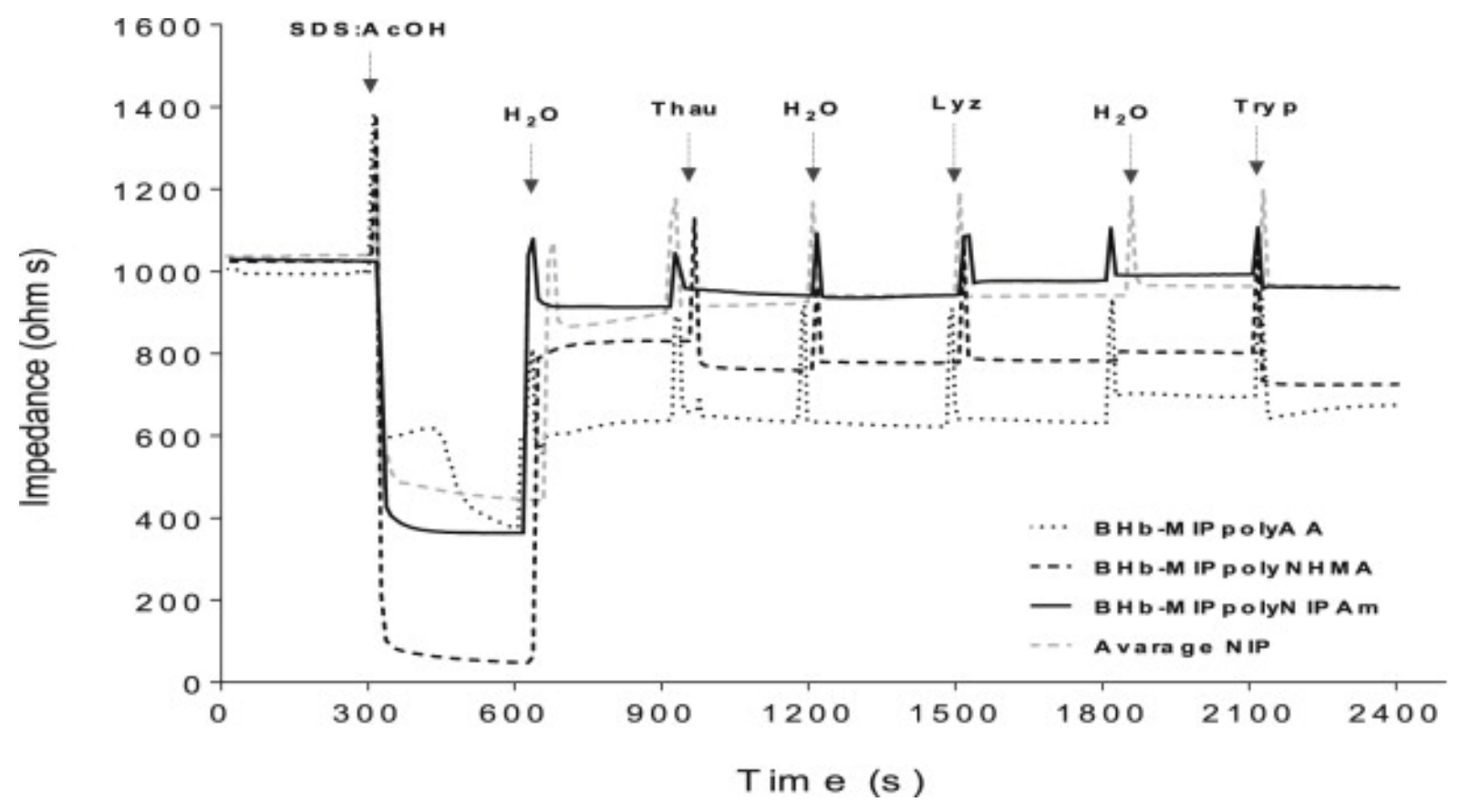

501

502

503

504

505

506

507

508 